\title{
A generalized fitting tool for analysis of two-dimensional channeling patterns
}

\author{
E. David-Bosne ${ }^{\mathrm{a}}$, U. Wahl ${ }^{\mathrm{a}, \mathrm{b}, *}$, J.G. Correia ${ }^{\mathrm{a}}$, T.A.L. Lima ${ }^{\mathrm{b}, 1}$, A. Vantomme ${ }^{\mathrm{b}, 1}$, \\ L.M.C. Pereira ${ }^{b, 1}$ \\ ${ }^{a}$ Centro de Ciências e Tecnologias Nucleares (C2TN), Departamento de Engenharia e Ciências \\ Nucleares, Instituto Superior Técnico, Universidade de Lisboa, 2695-066 Bobadela, Portugal \\ ${ }^{\mathrm{b}}$ KU Leuven, Instituut voor Kern- en Stralingsfysica, 3001 Leuven, Belgium \\ \# Current address: KU Leuven, Quantum Solid-State Physics, 3001 Leuven, Belgium \\ *Corresponding author, email address: uwahl@ctn.tecnico.ulisboa.pt (U. Wahl)
}

\begin{abstract}
The acquisition of two-dimensional (2D) channeling patterns is gaining increased popularity within the ion beam community. However, with the exception of emission channeling experiments for the lattice location of radioactive impurities, quantitative analysis of such patterns is rarely found. We present a general description of the statistical data analysis methodology for 2D channeling patterns, which consists of comparing experimental data by means of a fit procedure to theoretical yield distributions. The developed software allows for chi-square or maximum likelihood-based fits, optimizing the orientation of the theoretical vs the experimental pattern, as well as the best choice of random level, and providing fractions for the contributions from several theoretical patterns related to different lattice sites. Optionally also the angular resolution can be used as a fit parameter. Use of the software is illustrated by examples of electron emission channeling from ${ }^{27} \mathrm{Mg}$ in $\mathrm{GaN}$, as well as ${ }^{4} \mathrm{He}$ RBS channeling from $\mathrm{Ge}$.
\end{abstract}

Keywords: Two-dimensional channeling pattern, blocking pattern, image scan, positionsensitive detector, fitting software, RBS/C, emission channeling

\section{Motivation: the use of two-dimensional patterns in channeling measurements}

The channeling effect of highly energetic charged particles in single crystals is a wellestablished phenomenon that finds it applications in a variety of ion beam techniques, e.g. for characterizing the quality of single crystals, assessment of ion implantation damage and its annealing, epitaxial relationships of thin films and substrates, or for lattice location studies of impurities [1-3]. In one way or another, channeling experiments always involve the measurement of particle yields as a function of angle towards major crystallographic directions, either as a function of the angle of an incoming beam, or as a function of particle exit angle. In most cases, particle yields are recorded in the form of so-called angular scans, i.e. one-dimensional (1D) functions of polar angle from major crystallographic directions at a fixed azimuth, or of azimuthal angle while keeping the polar angle fixed. However, there are increasingly more reports that supply complete two-dimensional (2D) data arrays of the quantity, so-called channeling or blocking patterns, which provide considerably more detailed information than simple 1D angular scans. The methods to obtain 2D channeling patterns vary depending on whether channeling effects occur for an incident particle beam or for outgoing particles. For instance, in Rutherford Backscattering Spectrometry/Channeling $(\mathrm{RBS} / \mathrm{C})$ [1-2] experiments a beam of protons or helium ions, which impacts on a single crystal, is used as the source of channeled particles. In most RBS/C applications, the ion beam is directed towards the single crystal under small angles to or aligned with major 
crystallographic directions, so that the channeling effect occurs on the inward path of the particles. Particles which were backscattered in close collisions with lattice or impurity atoms are then detected by conventional non position-sensitive detectors, either in point-like geometry or with a large solid angle, depending on whether kinematic accuracy (and related depth resolution) or detection efficiency need to be optimized. For 2D channeling patterns to be obtained by using this experimental approach, the ingoing beam angle is generally varied in equidistant $x$ - and $y$-steps ( $\theta$ and $\omega$ angles) around the channeling direction, a procedure which unfortunately has been given various names in the literature, e.g. "image scan" [4-6], "angular resolved channeling image" [7], "three-dimensional visualization of ion channeling " [8], "two-dimensional channeling pattern" [9], "angular scan image" [10], "angular yield maps" [11], "angular dependent ion channeling" [12], "angle-resolved scan" [13], "full scan maps" [14], "RBS-C maps" [15], "channeling crystal image" [16], "channeling image" [17], "angular resolved RBS data" [18], "2D ion channeling image" [19], "channeling/blocking pattern" [20], and "angular scan mapping" [21]. While such type of 2D scans are used by numerous groups, e.g. in order to precisely orient samples along channeling directions or to powerfully visualize the channeling effect, detailed quantitative data analyses based on the acquired patterns are only rarely found in the literature, e.g. in Refs. [5, 12, 14, 18]. Despite the fact that 2D scans contain a wealth of information and take quite some effort to record, one of the reasons that their detailed analysis is not more widespread may be that suitable computer programs which allow quantitative analysis of $2 \mathrm{D}$ channeling data are not commonly available.

In a different approach, the ion beam is sent into the crystal in a non-channeling, "random" direction, and the channeling and blocking effects of the emerging particles on their way out of the crystal are detected. In this case, while the ingoing angle is kept fixed, the $2 \mathrm{D}$ patterns of outgoing particles can be conveniently recorded with the help of position-sensitive detectors (PSDs). While this approach is also not common, applications have been reported in the literature using RBS/C [22-25], Medium Energy Ion Scattering (MEIS) [26-30] or Elastic Recoil Detection Analysis (ERDA) [31-32].

An example outside the field of RBS/C but where routinely 2D channeling patterns from PSDs are used, and which motivated the work presented here, are emission channeling (EC) lattice location studies. EC uses implanted radioactive isotopes and the channeling effects are measured from the charged particles emitted during nuclear decay [33-37]. Most commonly $\beta^{-}$particles or conversion electrons (CEs) are used due to the wide choice of suited isotopes but applying $\alpha[35,38]$ or $\beta^{+}$emitters [33] is also possible. In EC experiments $2 \mathrm{D}$ channeling patterns of outgoing particles are recorded with the use of various types of PSDs [36, 39-41]. The obtained information on impurity lattice sites and possible site changes, e.g. with sample annealing temperature, is of interest for the understanding of electronic, magnetic and optical properties of materials that depend on the lattice site of dopants. The key advantages of EC in comparison to RBS/C lattice location techniques are roughly four orders of magnitude higher efficiency and the ability to study also elements lighter than the host atoms; the major limitation is that a production facility for radioactive ion beams is needed, while RBS/C experiments can be performed at more commonly available stable ion beam facilities.

While the theoretical approaches for describing the channeling of $\beta$ or $\alpha$ particles are quite different, the final step for the analysis of experimental channeling data, both from RBS/C and $\mathrm{EC}$, is similar and consists in fitting the experimental 1D scans or 2D patterns by a linear combination of theoretical angular yield distributions calculated for impurities on different lattice sites, under assumptions that match the experimental conditions as closely as possible. For the quantitative analysis of $2 \mathrm{D}$ emission channeling patterns, chi-square fit routines called FDD (short for "Fit in 2 dimensions") have been briefly reported first in 1996 [38], 
followed by further remarks in Refs. [36, 39-40]; they were, however, never documented in the literature in detail. With the recent exploration of Timepix detectors [42] (a highly pixelized PSD developed by the Medipix collaboration [43]) for emission channeling experiments, it was necessary to review the fitting methods used in order to fully take advantage of the spatial resolution of the detector and overcome the problem of having a reduced number of counts per pixel in the chi-square fit. For that purpose, new software called PyFDD (short for "Python FDD") has been developed. As an improvement over the predecessor FDD, it is optimized for measurements with a low number of counts per pixel, which is a common situation when using modern PSDs with a high pixel density. At the same time PyFDD is also adequate for the analysis of measurements with PSDs consisting of large pixels, where the pixel yield needs to be modelled as the integral of the simulation over the pixel area.

This paper is intended as a general description of the statistical data analysis methodology for lattice location experiments using two-dimensional channeling patterns. We will describe both the approach chosen in our older software FDD, as well as the one implemented in the new PyFDD. Although the methods and software presented were developed for the emission channeling technique, they can, with due care, also be applied e.g. to the analysis of 2D RBS/C patterns. Providing the newly developed PyFDD as an open source software [44] makes it directly available to potential other users.

\section{Emission channeling methodology}

In an electron emission channeling experiment, a single crystal is implanted with a radioactive isotope that emits beta particles or conversion electrons. Depending on the lattice site of the emitting atom, the emitted particles experience channeling or blocking effects that are prominently visible along the major crystallographic axes and planes. The effects are measured as the anisotropy of the electron emission yield along different angular directions using a PSD (Fig. 1). Various key aspects of the technique have been described in detail previously: ISOLDE/CERN as the radioactive beam facility [45-46], the implantation and measurement chamber [47], the position sensitive electron detectors [36, 40-41], and the "manybeam" simulation software for electron channeling [33, 36]. The fitting software makes the connection between the data from the detector and the results of many-beam simulations: the angular anisotropy is measured as a 2D histogram of counts, where each bin represents the counts in a pixel which covers an area of specific angular range with respect to the crystal axis; for quantitative analysis, the 2D histograms, also called patterns, are then compared to simulated data using a fitting routine.

The major types of PSDs that have been used so far in ion beam and emission channeling experiments, are Si resistive charge division detectors [22, 24, 34-35], ionization chambers [31-32], Si pad detectors [39-40], multi-channel plates [23, 26-30], as well as Timepix pixel detectors [25]. Each detector system has specific characteristics that influence the observed channeling patterns and need to be accounted for in the data analysis procedures. Characteristics to keep in mind are the energy resolution, which is especially important for experiments that require the selection of an energy region of interest (ROI), e.g. for ion channeling or conversion electron emission channeling; the energy threshold of particle detection that is commonly related to electronic noise; and the detection efficiency, which should be uniform over the whole area of the detector. Since X-rays or gammas form an undesired source of background, low detection efficiency for photons is highly desirable. The maximum achievable count rate is also an important aspect as high throughput allows reducing experimental time while keeping detector dead time (and corresponding additional radiation damage to the sample) and pile-up artefacts to a minimum. However, the most 


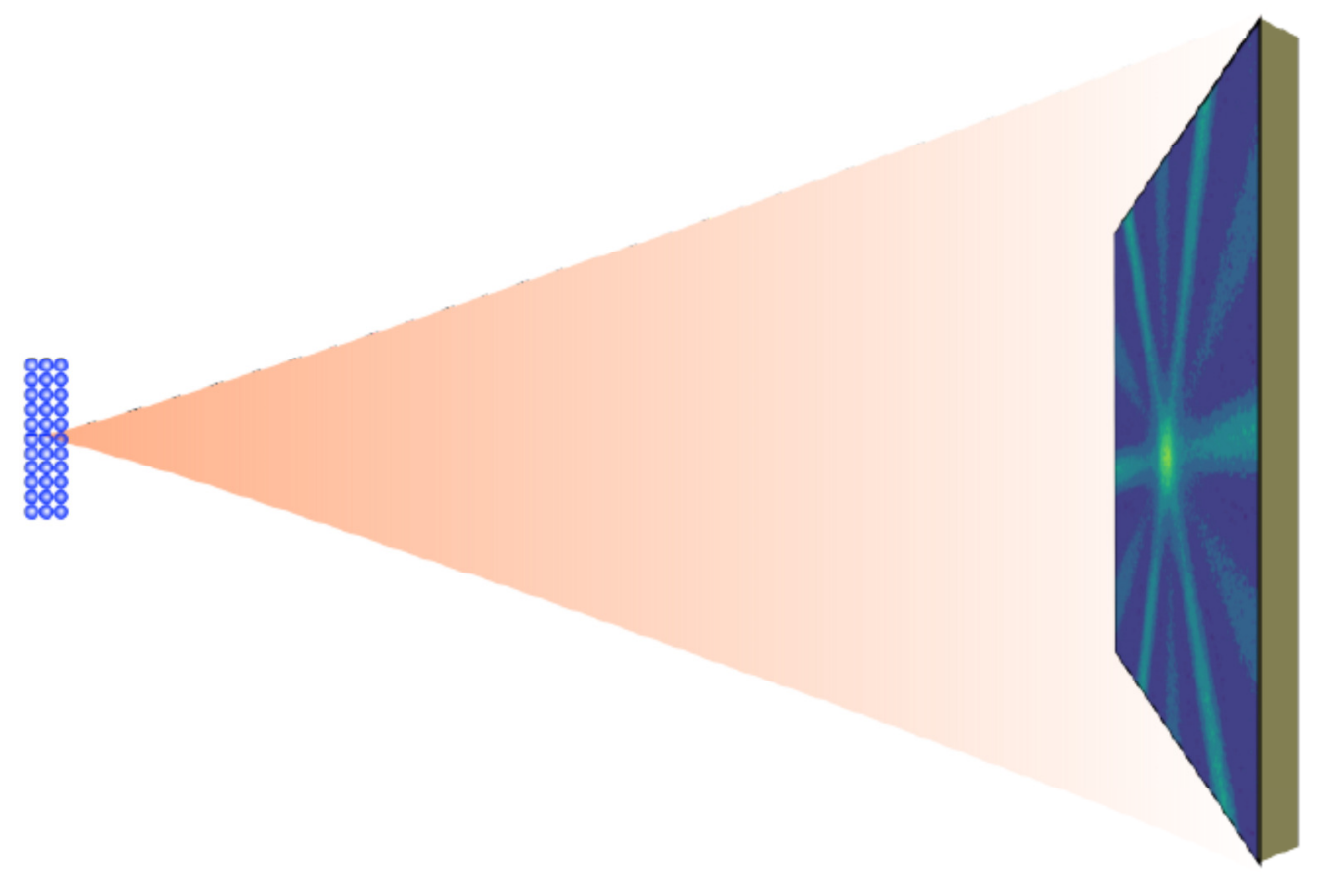

Fig. 1. Schematic illustration of the emission channeling method. The channeling effects of charged particles emitted from radioactive probe atoms inside a single crystal are measured using a position-sensitive detector. In this image the brighter yellow regions of the detector represent a higher electron emission yield along crystallographic axes and planes. The shape and intensities that describe the pattern reflect the crystallographic symmetry of the crystal as well as the lattice sites occupied by the emitter atoms and the respective site fractions.

crucial feature of a PSD is its position resolution, as will become clear by the following considerations.

One of the main characteristics of any channeling experiment is the angular resolution, which depends on the measurement geometry. When using a PSD the emission angle assigned to a detected particle is derived from the angle between the crystallographic direction and the straight line that connects the beam spot with the position where the particle was registered in the detector. The angular resolution is then intrinsically limited by the uncertainty in the particle origin related to the beam spot dimension (often $\sim 1 \mathrm{~mm}$ diameter) and the uncertainty in the hit location on the detector due to its limited position resolution. If these two contributions to the angular resolution are considered as Gaussian distributions, the total effect on the pattern can be simplified as the convolution with a single Gaussian of standard deviation $\sigma_{\text {total }}$ (in units ${ }^{\circ}$ )

$\sigma_{\text {total }} \approx \frac{\sqrt{\sigma_{\text {detector }}^{2}+\sigma_{\text {beamspot }}^{2}}}{d} \times \frac{360^{\circ}}{2 \pi}$

where $\sigma_{\text {detector }}$ and $\sigma_{\text {beamspot }}$ (in units of length) are the standard deviations related to the position resolution of the detector and the beam spot distribution, and $d$ is the distance between sample and detector. The values for each contribution are usually known only approximately: the PSD position resolution depends on particle species and energy; the 
distribution of the beam spot on the sample depends on the beam profile after collimation and the angle of implantation. Moreover the projection of the beam spot on the sample changes with angle of measurement. Additional uncertainty can be caused by imperfections of the crystallographic structure of the sample itself, e.g. if crystallographic directions show angular spread due to mosaicity [48], i.e. the crystal has domains that are slightly tilted or rotated from each other, the measured channeling effects may consist of a superposition from various domains.

Taking into account the position resolution of a specific detector, different choices can be made on how to bin the angular yield data into a histogram during data taking and/or analysis. For example, while the position signal from a resistive charge division detector is a continuous variable, after digitalization it is often sorted into $64 \times 64$ or $128 \times 128$ bins since spatial resolution is usually around several per cent $[22,24,35]$. This provides the advantage of having a high number of counts per bin without significantly losing position resolution. For the Si pad detectors so far used in electron emission channeling [39-40], which have $22 \times 22$ pixels of size $1.3 \times 1.3 \mathrm{~mm}^{2}$, the resolution limit is given by the large pad size, so each detector pad corresponds to a histogram bin. With the introduction of the Timepix PSD [42] and its good position resolution due to the $512 \times 512$ pixels of size $55 \times 55 \mu^{2}$, the data binning had to be re-assessed, taking into account a trade-off between the number of counts per bin necessary for a chi-square fit, and the position resolution. High angular resolution should be beneficial for cases of lattice location of radioactive probes with high-energy beta spectra and for increased sensitivity on changes in crystal quality during the course of the experiment. Particular benefit is expected for short-lived isotopes with mean beta energies $<E_{\beta}>$ above $1.5 \mathrm{MeV}$, e.g ${ }^{11} \mathrm{Be}\left(t_{1 / 2}=13.8 \mathrm{~s},<E_{\beta}>=4.65 \mathrm{MeV}\right),{ }^{19} \mathrm{O}(26 \mathrm{~s}, 1.74 \mathrm{MeV})$, or ${ }^{6} \mathrm{He}$ $(807 \mathrm{~ms}, 1.57 \mathrm{MeV})$. In this paper, examples using the isotope ${ }^{27} \mathrm{Mg}(9.46 \mathrm{~min}, 0.70 \mathrm{MeV})$ will be addressed.

\section{Channeling simulations}

In order to quantitatively analyze $2 \mathrm{D}$ channeling patterns, these need to be compared to theoretical yield distributions by means of a fit procedure. Since the required channeling simulations are rather time-consuming (several months of computation may be required), performing them in advance prior to data fitting is indispensable. The details on the formalisms for channeling simulations can be found in Ref. [34, 36] for electron EC, in Ref. [49] for RBS/C, and in Ref. [35] for $\alpha$ EC. Aspects such as the choice of interaction potentials, the crystallographic structure, the displacement of host atoms due to thermal vibration, and appropriate models for dechanneling need to be chosen carefully. Simulations usually start from beams of particles entering into the crystal from the outside under a small angle or aligned with a channeling direction. While this corresponds to the real experimental situation for RBS/C 2D image scans, it is not the case when measuring the channeling of outgoing particles by means of PSDs. However, due to the Lindhard reciprocity theorem [50], which is based on the time reversal of trajectories, the channeling of outgoing particles is equivalent to the computational approach with an ingoing beam of particles (as long as energy losses are small) [33, 35-36]; however, for programming purposes the latter is much simpler to realize. Depending on the nature of the projectiles, different theoretical approaches are used to describe the movement of charged particles inside a crystal. Ion channeling is modeled by Monte Carlo simulations based on trajectories derived from classical mechanics see e.g. [49], while electron channeling is treated by the quantum-mechanical "many beam" model [33, 36], which involves finding the solution to a Schrödinger equation in a periodic potential by means of Bloch waves. Both approaches allow calculating the flux density of channeled particles at given lattice sites as a function of depth, which is the most time 
consuming part of the simulations and needs to be performed up to the maximum expected depth of probe atoms. Results are then convoluted with the probe atom depth profile, which in case of implanted probes is estimated from SRIM simulations [51]. In case of $\beta^{-}$EC, due to the continuous nature of $\beta^{-}$spectra, the simulations are performed for a wide range of electron energies and the results weighted according to the energy distribution of the $\beta^{-}$ spectra. Simulations typically cover an angular range of $\pm 3^{\circ}$ around the channeling axis in a mesh of $0.050^{\circ}$ or $0.025^{\circ}$ bins, so that each lattice site is characterized by a $2 \mathrm{D}$ angular yield pattern of $121 \times 121$ or $241 \times 241$ bins. Nowadays it is common practice to simulate patterns for all substitutional as well as the major interstitial sites in the crystal, plus vector displacements in between them in steps of $\sim 0.05 \AA$. In a cubic crystal, this corresponds to displacements from the substitutional sites along $<111>,<100>$ or $<110>$ directions. Presently, several hundred patterns corresponding to simulations for all desired lattice sites are packed in a library file for subsequent use by the fitting software.

The patterns in the library are given in terms of "normalized yield" $\chi$, which is a key concept for how the channeling effect is treated theoretically. Normalized yields are defined relative to the yields that would be measured from a sample devoid of any channeling effects, which is straightforward to implement in the theoretical approaches while experimentally this would correspond to measuring the yield from an amorphous sample under exactly the same conditions as the single crystal, which is never feasible. As an example, let us assume a situation where we have three fractions $f_{1}, f_{2}, f_{3}$ of emitter atoms on lattice sites $1,2,3$. If $\chi_{1}(\theta, \varphi), \chi_{2}(\theta, \varphi), \chi_{3}(\theta, \varphi)$ are the theoretically calculated normalized yields for these sites under angles $\theta, \varphi$ from the channeling axis, the expected number of counts $N(\theta, \varphi)$ measured is then given by the basic relation

$\frac{N(\theta, \varphi)}{N_{\mathrm{R}}}-1=f_{1}\left[\chi_{1}(\theta, \varphi)-1\right]+f_{2}\left[\chi_{2}(\theta, \varphi)-1\right]+f_{3}\left[\chi_{3}(\theta, \varphi)-1\right]$

where $N_{\mathrm{R}}$ is the number of events that would be measured in case of an amorphous sample and in channeling terminology is called the "random level". Note that due to the way the theoretical yields are defined, there is no way to analyze experimental channeling data without making a choice, either explicit or implicit, for $N_{\mathrm{R}}$. When analyzing $2 \mathrm{D}$ channeling patterns, it is usually chosen to obtain the normalization from the fit procedure which compares experimental and theoretical patterns, as will be outlined below.

\section{Data fitting software and methodologies}

This section will go through the data analysis processes of building statistical estimators for lattice site occupancy fractions in the FDD software and its new python alternative PyFDD. Most of the methods described are applications of well-documented fit concepts, cf for instance Ref. [52], and it will be specifically mentioned when non-standard approaches were implemented.

The initial fit software, called FDD was programmed in FORTRAN in the 1990s by Wahl [36, 38-40] and ran under DOS but used already a graphical user interface. Later the same methodology and mathematical routines were transferred to Java by De Vries [53]; a major upgrade by Amorim [54] added functionalities that allow the execution of macros and conveniently perform fits for a whole series of site combinations. Both the older FDD versions and PyFDD have a similar overall approach to data fitting, illustrated in Fig. 2, although the specifics of the methods are different. In the analysis it is necessary that the fitting methods provide estimates for the model parameters, and if possible also confidence intervals for these parameters and feedback on goodness of fit. 
The first step when building a fit function from the simulated patterns is to decide which sites to include in the fit. Although the software can handle more sites, in practice it is found that usually only the two most frequently occupied sites can be identified, while statistically meaningful three-site analyses were possible only in some special cases. Once sites are chosen, their corresponding angular yield patterns $\chi_{1}, \chi_{2}, \chi_{3} \ldots$ are linearly combined, together with a flat pattern (a matrix of ones) representing so-called "random" sites, an approach that becomes obvious once eq. (1) is rearranged as follows:

$N(\theta, \varphi)=N_{\mathrm{R}} \times\left[f_{1} \chi_{1}(\theta, \varphi)+f_{2} \chi_{2}(\theta, \varphi)+f_{3} \chi_{3}(\theta, \varphi)+1-f_{1}-f_{2}-f_{3}\right]$

The "random fraction" $f_{\mathrm{R}}=1-f_{1}-f_{2}-f_{3}$ corresponds to the fraction of emitter atoms not causing any anisotropy in the patterns because they are located in amorphous regions of the sample or on low-symmetry lattice sites. However, any flat background in the pattern from other sources, e.g. from backscattered electrons or gamma background in the detector, will also be forced into the random fraction which then has to be appropriately corrected. Note that the $f_{\mathrm{i}}$ are not equivalent to estimating the fraction of measured particles that originated from each site. For example, assuming identical occupancy fractions, a pattern that shows a strong channeling peak will contribute with more particles to the measured pattern than one showing a blocking dip.

\subsection{FDD fitting method}

FDD is the software traditionally used for fitting EC patterns and will be shortly described here, also as an introduction to PyFDD. FDD estimates the model parameters by using a Levenberg-Marquardt routine [55] to iteratively minimize a chi-square function. Since the concept of FDD dates back to the early 1990s, when computer power was rather limited, a number of features were implemented in a way that traded statistical accuracy for simplicity, still resulting in a code that is both fast and robust in estimating model parameters from channeling patterns.

The first step in FDD is to divide the integer number of observed counts $N_{i}$ measured in each bin $i$ by a first guess $N_{\text {Rguess }}$ for the number of counts $N_{\mathrm{R}}$ that would be measured without any channeling effects, i.e. in a totally random direction of the sample. $N_{\text {Rguess }}$ is estimated from the measured data by the user by averaging the number of counts over a range of pixels that do not contain major channeling effects. At this point each bin corresponds to a detector

pixel. The data bin values become thus real numbers $\chi_{i}^{\text {experiment }}$, representing relative yields for each angular position

$\chi_{i}^{\text {experiment }}=\frac{N_{i}}{N_{\text {Rguess }}}$

The standard deviation $\sigma_{\mathrm{i}}$ for the yield in each data bin is calculated as the square root of the observed value $N_{\mathrm{i}}$ scaled by $N_{\text {Rguess }}$

$\sigma_{i}=\frac{\sqrt{N_{i}}}{N_{\text {Rguess }}}$

Calculating the standard deviation from the square root of detected counts means that areas with a high count rate, as from axial or planar channeling effects, are statistically somewhat more relevant for the fit results than e.g. blocking dips, and also that pixels with zero counts have to be excluded from the fit. Using the variances $\sigma_{i}^{2}$ of experimental data results in the Neyman chi-square method which has an increased bias over the Pearson least square where the yield of the theoretical model is used as expected variance. The fact is that both Pearson's and Neyman's chi-square approaches are biased, as the first will over-estimate the volume under the curve and the latter will underestimate it [56-57]. However, the difference between 
both methods is only significant in cases where the number of counts per pixel is low, as is the case when using high pixel density detectors like Timepix.

Since for the pad detectors typically used in EC, the detector pixel size of $1.3 \mathrm{~mm}$ corresponds to an angular range of $\sim 0.25^{\circ}$, which is much larger than the simulation steps of $0.025^{\circ}-0.05^{\circ}$, the fit procedure needs to average over the range of simulated yields. In order to approximate this by a fast computational procedure, it is usually chosen during FDD fitting of pad detector data to divide each pad in a finer mesh of $6 \times 6$ bins with equal $\chi_{i}^{\exp }$ and $\sigma_{\mathrm{i}}$ values. While this guarantees a fast averaging process, it has the disadvantage that the significance of the resulting chi-square as a statistical indicator for goodness of fit is distorted. We will come back to this point later.

In order to account for the angular resolution resulting from the size of the beam spot, FDD uses simulation libraries with patterns that have been smoothed by convoluting with Gaussians of standard deviation $\sigma_{\text {conv }}$; usually values for $\sigma_{\text {conv }}$ are chosen as integer multiples $(0,1,2,3 \ldots)$ of the simulation bin size of $0.025^{\circ}$ or $0.05^{\circ}$. As the smoothing is done prior to fitting, only the available discrete values for $\sigma_{\text {conv }}$ may be applied and a fine variation of $\sigma_{\text {conv }}$ during fitting is not possible.

A linear combination of the selected smoothed patterns is used to create the model function that is to be optimized by the fit routine, where the expected pattern $\chi_{i}^{\text {expected }}$ is defined as

$\chi_{i}^{\text {expected }}=S \times\left[f_{1} \chi_{1 i}\left(\sigma_{\text {conv }}\right)+f_{2} \chi_{2 i}\left(\sigma_{\text {conv }}\right)+f_{3} \chi_{3 i}\left(\sigma_{\text {conv }}\right)+\left(1-f_{1}-f_{2}-f_{3}\right)\right](6)$

$S=N_{\mathrm{R}} / N_{\text {Rguess }}$ is a scaling parameter that fine tunes the initial normalization and thus accounts for the fact that the guessed number of events per pixel in a random part of the pattern $N_{\text {Rguess }}$ deviates (usually by only a few $\%$ ) from the number $N_{R}=S N_{\text {Rguess }}$ that the fit finds best. Note that chi-square fitting of histograms with a common scaling factor as fit parameter is known to introduce a bias on the results, meaning that the sum of the fit residues does not average to zero [56-57]. While this problem is negligible when the number of counts is high (above a few hundred per bin), for fitting patterns with low statistics in FDD it can only be circumvented by keeping $S$ at a carefully chosen fixed value.

For the next step the expected pattern is rotated by an azimuthal angle $\varphi_{0}$ and translated by $\left(x_{0}, y_{0}\right)$ as indicated in Fig. 2 in order to match the measurement orientation. As this is done in a similar way in PyFDD the description can be found in the next section. The fitting process then minimizes a reduced chi-square function defined as

$\chi_{\text {red }}^{2}=\frac{1}{N_{\text {dof }}} \sum_{i=1}^{N_{\text {bins }}} \frac{\left(\chi_{i}^{\text {experiment }}-\chi_{i}^{\text {expected }}\right)^{2}}{\sigma_{i}^{2}}$

where $N_{\text {bins }}$ is the total number of bins or pixels used in the fit, and the number of degrees of freedom $N_{\text {dof }}$ is equal to $N_{\text {bins }}$ minus the number of fit parameters. Dividing the chi-square by $N_{\text {dof }}$ is commonly thought as a rule of thumb method for estimating the goodness of fit with the value approaching unity for good fits. However, when fitting pad detector data with the procedure described above, due to the fact that $N_{\text {bins }}$ has been multiplied by 36 when dividing the large pixels into smaller bins, reduced chi-square values are even for high-quality fits several times larger than one. While the fit results are still meaningful, the ability to use the resulting chi-square for further statistical inference is lost. 


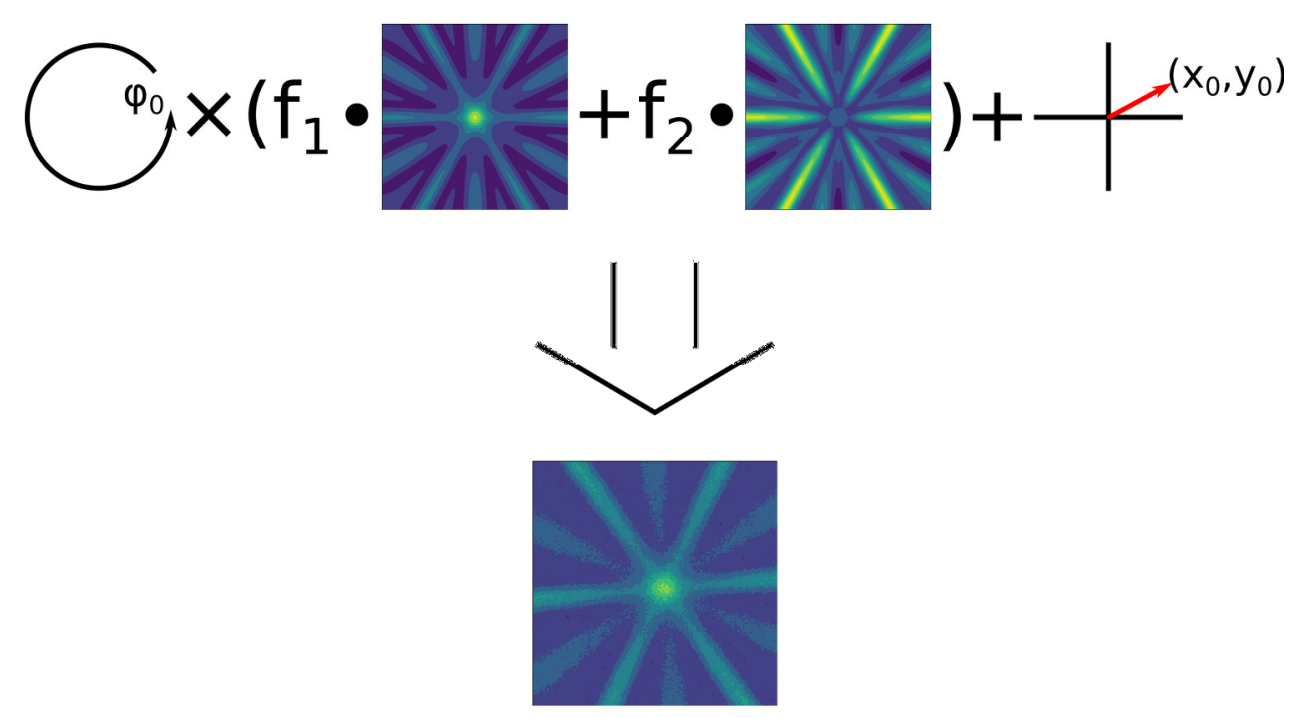

Fig. 2. Schematic illustration of the fit approach used to most accurately describe the patterns seen by the measurement. First, a number of lattice sites are chosen, usually not more than two, as is shown in this figure. A linear combination of their simulated angular yield patterns is then formed using site fractions $f_{1}$ and $f_{2}$, plus the contribution $1-f_{1}-f_{2}$ from a flat pattern (not shown). The resulting combined pattern is rotated by an angle $\varphi_{0}$ and translated to $\left(x_{0}, y_{0}\right)$ in order to match the orientation of the crystallographic axis with respect to the detector. The fractions $f_{1}$ and $f_{2}$ as well as the geometrical adjustments through rotation and translation are iteratively optimized by the fit routine.

\subsection{PyFDD fitting methods}

This section explains how the new software PyFDD builds the model function, calculates the cost function and provides tools for error estimation. PyFDD uses standard methods building the model function as a probability density function (p.d.f.) and using unmodified formulas for the cost functions. The software provides two choices for the cost function, chi-square and maximum likelihood; both use the p.d.f. defined in the same way. The minimization algorithm for both cost functions is the L-BFGS-B from the Scipy python library [58] which uses a limited memory (L) and bounded (B) version of the Broyden-Fletcher-GoldfarbShanno (BFGS) algorithm. This algorithm allows setting limits to parameters that are bound by physical constraints, thus preventing, e.g. that lattice site occupancy fractions become negative or excessively large.

\subsubsection{Building the probability density function}

For fitting channeling patterns the model is represented by a 2D p.d.f. that depends on various parameters, including those that are actively optimized by the fit. By definition the p.d.f. is normalized to a sum of one, thus representing for each given $(x, y)$ bin position the probability that a particle is measured at that location when the detector registers one event. Its parameters are, as defined by the physical model, the lattice sites, the occupancy fractions $f_{\mathrm{i}}$, the Gaussian convolution sigma $\sigma_{\text {conv }}$ that takes into account the effects expressed in eq. (1), and experimental conditions such as the $\left(x_{0}, y_{0}\right)$ position of the channeling axis center and the azimuthal rotation angle $\varphi_{0}$. To simplify the following formulae, we will use the variables $P$ to symbolize theoretical patterns, and $E$ as expected and $O$ as observed experimental results. 
First, the calculated patterns for all chosen sites are added with the corresponding fractions in order to obtain $P_{\text {ideal, }}$, the theoretical pattern combining several sites with ideal angular resolution

$P_{\text {ideal }}\left(f_{1}, f_{2}, f_{3}\right)=f_{1} P_{1}+f_{2} P_{2}+f_{3} P_{3}+\left(1-f_{1}-f_{2}-f_{3}\right) \times P_{\text {rand }}$

where $P_{\text {rand }}$ is a matrix of ones. The next step is to apply a Gaussian convolution

$P\left(f_{1}, f_{2}, f_{3}, \sigma_{\text {conv }}\right)=$ GaussConv $\left[P_{\text {ideal }}\left(f_{1}, f_{2}, f_{3}\right), \sigma_{\text {conv }}\right]$

The strategy of finding the best $\sigma_{\text {conv }}$ may be either to fix it based on a geometrical estimate or to consider it as a parameter to be optimized during the fit. The pattern is then azimuthally rotated by an angle $\varphi_{0}$ and translated with variables $\left(x_{0}, y_{0}\right)$ to match the orientation of the experiment. The next step is to interpolate the simulation values at the coordinates of the detector pixels. For detectors that have large pixels, it is chosen to interpolate a grid of points equally spaced inside each pixel ( $6 \times 6$ for pad detectors) and average the results.

Finally the p.d.f. is built by normalizing the resulting pattern $P$ in order to yield an integral equal to one

p.d.f. $\left(f_{1}, f_{2}, f_{3}, x_{0}, y_{0}, \varphi_{0}, \sigma_{\text {conv }}\right)=\frac{P\left(f_{1}, f_{2}, f_{3}, x_{0}, y_{0}, \varphi_{0}, \sigma_{\text {conv }}\right)}{\sum_{i} P_{i}}$

where $\sum_{i} P_{i}$ is the sum over all bins used in the fit, excluding e.g. masked pixels that are known to have a bad response.

\subsubsection{Chi-square fit}

PyFDD follows the principle of not modifying at all the experimental data to be fitted. Thus, instead of scaling the observation to the model, a p.d.f. is built which is then scaled by multiplying it with the sum of all events $N_{\text {cts }}$ included in the experimental pattern

$E\left(f_{1}, f_{2}, f_{3}, x_{0}, y_{0}, \varphi_{0}, \sigma_{\text {conv }}, N_{\text {cts }}\right)=N_{\text {cts }} \times p . d . f .\left(f_{1}, f_{2}, f_{3}, x_{0}, y_{0}, \varphi_{0}, \sigma_{\text {conv }}\right)$

Comparing eq. (11) to eq. (3) one sees that in this case the relation

$N_{\mathrm{R}}=\frac{N_{\mathrm{cts}}}{\sum_{i} P_{i}}$

holds, i.e. the random level $N_{\mathrm{R}}$ is implicitly defined from the particular site distribution and the related theoretical patterns used in the fit. Note that since in typical channeling patterns an increase in yield within a peak is compensated by a decrease in nearby valleys, the quantity $\sum_{i=1}^{N_{\text {bins }}} P_{i}$ is therefore usually quite close to $N_{\text {bins }}$ and approximately $N_{\mathrm{R}} \approx N_{\text {cts }} / N_{\text {bins }}$.

Since the fit function has been scaled to the number of events, it is directly usable for calculating the variances $\sigma_{i}$ to be used in the Pearson chi-square as

$\sigma_{i}^{2}=E_{i}$

The Pearson chi-square is then calculated as

$\chi^{2}=\sum_{i=1}^{N_{\text {bins }}} \frac{\left(O_{i}-E_{i}\right)^{2}}{E_{i}}$

Note that this procedure decouples the variance used in the fits from the number of measured counts in a pixel, i.e. it does not a priori treat pixels that have measured a low number or zero counts as being statistically less relevant. Also, fitting without a scaling factor $S$ reduces biasing of the fit, especially when the overall number of events is low. Both aspects hence make this approach more appropriate to handle patterns with a low number of counts per bin. 


\subsubsection{Fit with maximum likelihood}

Chi-square has been the default method for the fit of channeling patterns. Its easy implementation and quick feedback over goodness of fit have made it a popular tool. However, as was mentioned already it is significantly biased when the number of counts per bin is low and especially when many bins contain zero events, which is not uncommon when performing emission channeling experiments with high density pixel detectors, such as the Timepix. Since the Timepix pixel size of $55 \mu \mathrm{m}$ is small compared to the beam spot size of $\sim 1 \mathrm{~mm}$, it is acceptable to use bins that are formed by the sum of several neighbouring pixels. However, while rebinning increases the number of counts per bin, thus allowing the use of the chi-square method, it progressively worsens the angular resolution. If chi-square is still not feasible after rebinning, the solution is to use the maximum likelihood method since it is unbiased. The maximum likelihood method finds the parameter model values for the p.d.f. that maximizes the probability of having sampled a distribution equal to the acquired data. Considering that the probability of an event occurring in bin $i$ is given by the p.d.f. value at the bin location, p.d.f. $\left(f_{1}, f_{2}, f_{3}, x_{0}, y_{0}, \phi, \sigma_{\text {conv }}\right)_{\text {i }}$, the total probability for the data set, called the likelihood, is given by

$L=\prod_{i=1}^{N_{\text {bins }}}\left[p . d . f .\left(f_{1}, f_{2}, f_{3}, x_{0}, y_{0}, \varphi_{0}, \sigma_{\text {conv }}\right)_{i}\right]^{o_{i}}$

where $O_{\mathrm{i}}$ is the number of counts measured in bin $i$. In order to simplify the computational optimization, instead of maximizing the likelihood function the negative of its logarithm is minimized. The used cost function is therefore the negative log likelihood function $-L L$, calculated as

$-L L=-\log (L)=-\sum_{i=1}^{N_{\text {bins }}} O_{i} \log \left[p . d . f .\left(f_{1}, f_{2}, f_{3}, x_{0}, y_{0}, \varphi_{0}, \sigma_{\text {conv }}\right)_{i}\right]$

The random level $N_{\mathrm{R}}$ in the experimental pattern is during maximum likelihood fitting also implicitly defined by eq. (12). As mentioned already, an advantage of parameter estimation using maximum likelihood is its robustness when bins have zero counts due to low statistics. While the terms relative to these bins in eq. (16) will be zero, the theoretical value $P_{\mathrm{i}}$ still contributes to the normalization and hence influences the random level through eq. (12). The disadvantage of the method is that the determination of the goodness of fit is not as direct as with the chi-square. For this reason chi-square is still a wise choice when counts per bin are high, typically above 50.

\subsection{Background correction in electron emission channeling}

Since electrons moving in matter are subject to pronounced scattering, it is impossible to measure electron emission channeling effects free from background of scattered electrons, in particular free from electrons that are backscattered from inside the sample or from the walls of the vacuum chamber. In addition, there is always a background from gammas emitted by the sample or from natural sources. All types of background usually contribute with a rather homogeneous count rate to the measured patterns, and will thus be contained in the random fraction $f_{\mathrm{R}}$ during a fit. The non-random fractions $f_{i}$ therefore need to be multiplied by a correction factor, which is equivalent to subtracting a flat baseline from the measured patterns.

The contribution of backscattered electrons is usually estimated by performing GEANT4 [59] Monte Carlo simulations of electron trajectories that take into account the main geometrical features and elemental composition of the sample, the sample holder, and the vacuum chamber, as well as the energy distribution of emitted $\beta^{-}$particles or conversion electrons. With $D_{\mathrm{e}}$ and $S_{\mathrm{e}}$ denoting the number of direct and scattered electrons in the GEANT4 simulations, the background correction factor $F_{\mathrm{e}}$ for electron backscattering is obtained as 


$$
F_{\mathrm{e}}=\frac{D_{\mathrm{e}}+S_{\mathrm{e}}}{D_{\mathrm{e}}}
$$

The background caused by gamma radiation emitted by the radioactive sample, as well as from natural radioactive sources and cosmic radiation, is usually experimentally estimated by closing a shutter in front of the detector, which stops all electrons from the sample while allowing through most of the $\gamma$ particles. With $\mathrm{C}_{\text {total }}$ and $\mathrm{C}_{\gamma}$ denoting count rates measured with shutter open and closed, the $\gamma$ background correction factor $\mathrm{F}_{\gamma}$ amounts to

$F_{\gamma}=\frac{C_{\text {total }}}{C_{\text {total }}-C_{\gamma}}$

The overall corrected site fractions $f_{i, \text { corrected }}$ are then given by

$f_{i, \text { corrected }}=F_{\gamma} \times F_{\mathrm{e}} \times f_{i}$

The absolute error in performing the background corrections as outlined above is estimated at $\pm 10 \%$.

\section{Statistical errors and systematic effects}

The statistical errors for the fit parameters are obtained from the Hessian matrix $H$ of the second partial derivatives of the cost function. In PyFDD, $H$ is not explicitly calculated during the cost function minimization routine, but by a separate routine after parameter optimization. The covariance matrix $C$ is then calculated from the Hessian as

$C=2 q H^{-1}$

where $q$ is a constant that is $q=1$ for chi-square and $q=1 / 2$ for maximum likelihood fitting [60]. The symmetric errors $\Delta x_{i}$ are obtained from the diagonal of the covariance matrix as

$\Delta x_{i}=\sqrt{C_{i i}}$

It must be pointed out that this procedure only allows quantifying the statistical errors of the fit parameters under the assumption that the model used is correct, which in particular means that the theoretical patterns used in the fit procedure correspond to actual emission patterns from the lattice sites that the probes are occupying. This, however, cannot be guaranteed. On the one hand, the assumptions underlying the calculation of the theoretical patterns may not be accurate. A known problem here is the effective depth distribution of probe atoms, which influences the intensity of channeling effects since electrons that are emitted from deeper inside the sample suffer more severe dechanneling. While dechanneling is implemented in the manybeam simulations, the procedure is approximate since it is based on a quantummechanical first-order perturbation theory. In addition, the assumed depth profile of probe atoms, which is usually taken from SRIM simulations [51] may be inaccurate, e.g. due to the fact that implanted probe atom depth profiles have channeling tails in the single crystal sample or are widened by diffusion during sample annealing. Dechanneling and insufficient knowledge of the probe atom depth profile are among the major sources of uncertainty which limit the quantitative analysis of electron channeling patterns. Another possible source of error are the root mean square (rms) displacements of the crystal host atoms which are an input to the manybeam simulations and influence both the crystal potential and the dechanneling. While rms displacements determined by X-ray or neutron diffraction at room temperature can be found for many materials in the literature, extrapolations to other temperatures usually involve approximations by means of a Debye model. Finally, the site combinations used in the fit may not correspond exactly to the sites that the probe atoms are occupying in the sample, meaning the used site model is not accurate or incomplete. As a rule, in EC experiments (and also in RBS/C) usually the statistical uncertainty in the fit 
parameters resulting from the fit is small compared to systematic effects. The best way to deal with this situation is to perform measurements for different crystallographic directions of the sample, in cubic samples commonly $\langle 111\rangle,\langle 100\rangle,\langle 110\rangle$ and $<211\rangle$ directions, analyze them separately and use the spread of the fitted site fractions and site locations in order to estimate systematic errors.

\section{Software}

PyFDD is an open source python library [44] and it is meant to be used in scripts. The structure of the program is illustrated by the dependency chart in Fig. 3 and described in more detail in the following.

The DataPattern class holds the measured data, orientation, mask for dead pixels and can perform some simple operations such as rotation, mirroring and cropping, it can also be saved in a Java Script Object Notation (JSON) file. The simulated patterns are handled by the PatternCreator class that uses the Lib2dl class to read the libraries and also produces the p.d.f. given the parameters $\left(f_{1}, f_{2}, f_{3}, x_{0}, y_{0}, \phi, \sigma_{\text {conv }}\right)$ for the required data bin locations. PatternCreator can also produce Monte Carlo generated patterns for testing. The Fit class handles the calculation and optimization of the cost function as well as statistical error estimation. The FitManager class calls a fit object for a given range of lattice sites and saves the results in a comma-separated value (.csv) file. A typical data analysis procedure starts by creating a DataPattern that is used to manually orient the sample and mask the necessary pixels. The DataPattern object is then saved into a JSON file. From FitManager one loads the

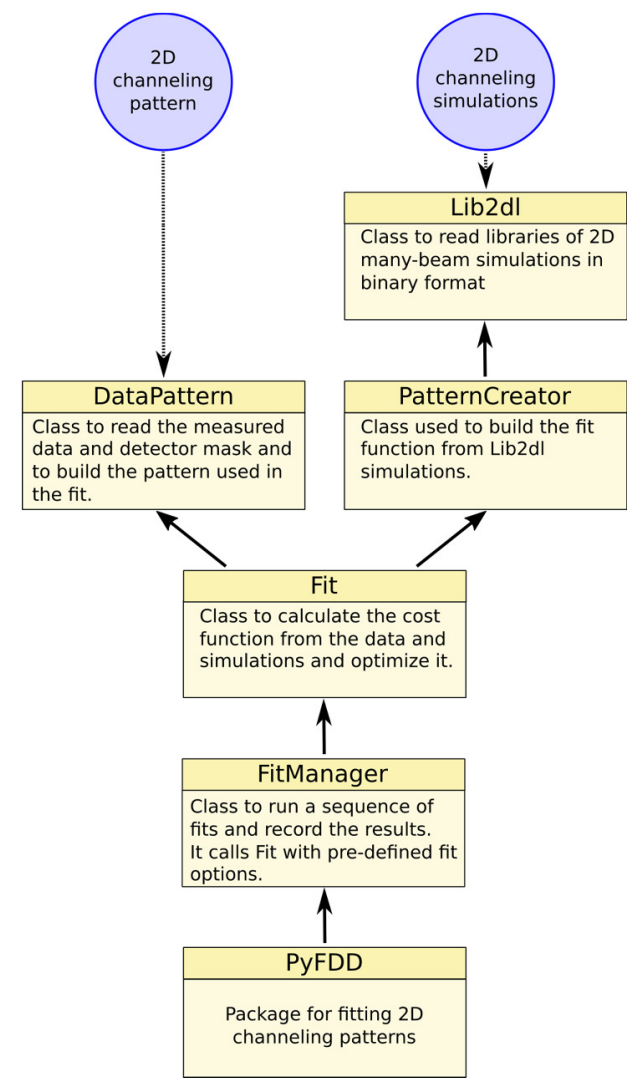

Fig. 3. Dependency chart of PyFDD modules and their description. In routine use the FitManager fits a range of lattice sites and exports the results in a table. While the other modules are called by FitManager they can also be used independently for specific cases. 
DataPattern file and performs all the necessary fits with a chosen range of lattice sites. Results are saved in a .csv file which can be analyzed by any spreadsheet and plotting software.

\section{Examples}

In this section, first two real case examples of the use of the PyFDD software for emission channeling are presented, followed by the analysis of an RBS/C pattern from a Ge single crystal. Both EC examples are for the analysis of [-1101] $\beta^{-}$emission channeling patterns from radioactive ${ }^{27} \mathrm{Mg}\left(t_{1 / 2}=9.46 \mathrm{~min}\right)$ in $p$-type $\mathrm{GaN}: \mathrm{Mg}$, however, one with the Timepix and the other with the pad detector. In case of the Timepix example, the $55 \mu \mathrm{m}$ sized pixels received typically only $\sim 4-5$ electrons for each measurement and therefore require a maximum likelihood approach in fitting. For the pad detector with its $1.3 \mathrm{~mm}$-sized pads, several thousand events per pad were recorded, consequently a chi-square approach is appropriate. The two experiments were performed with different samples and under different measurement geometries, which implies possible scatter in the results that is not due to effects of the detectors or analysis procedures.

The lattice location of ${ }^{27} \mathrm{Mg}$ in $\mathrm{GaN}$ is a well-known case which has been studied before [61], based on pad detector results analyzed with the older FDD software. While ${ }^{27} \mathrm{Mg}$ was found to occupy mainly substitutional Ga sites, a minority fraction on interstitial sites could be clearly identified. Based on analysis of [-1102], [-1101] and [-2113] patterns it was found that the interstitial $\mathrm{Mg}$ exhibits a displacement of $(+0.60 \pm 0.14) \AA$ from the ideal octahedral O position, i.e. it is located in between HA and HAB sites (Fig. 4). If only the [-1101] patterns

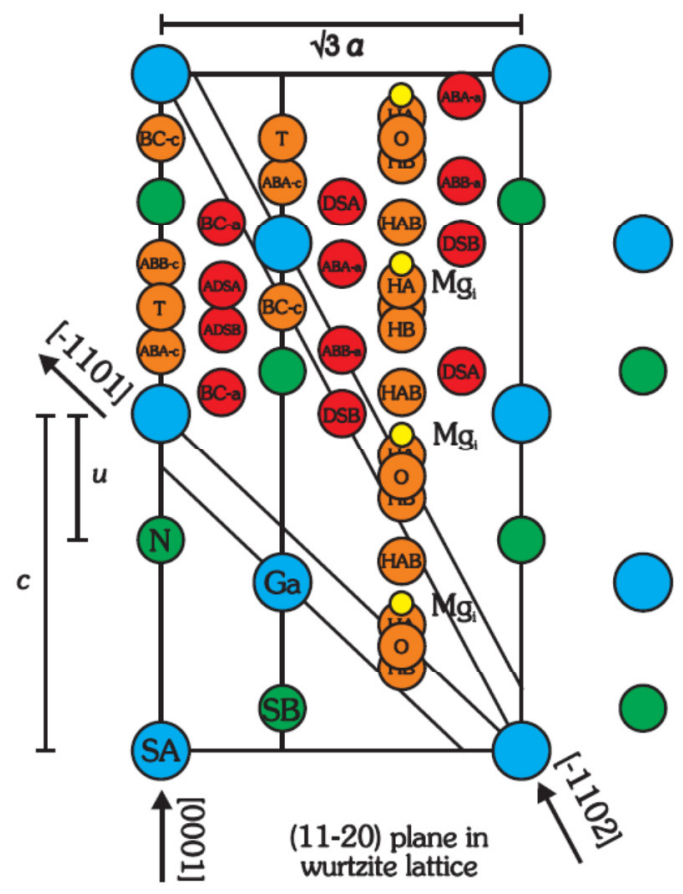

Fig. 4. Major substitutional and interstitial sites in the GaN wurtzite lattice. SA and SB represent the substitutional sites for $\mathrm{Ga}$ and $\mathrm{N}, \mathrm{BC}-\mathrm{c}$ and $\mathrm{BC}-\mathrm{a}$ are bond-centered sites along and off the $\mathrm{c}$ axis, $\mathrm{ABA}$ and $\mathrm{ABB}$ are antibonding sites, DSA, ADSA, DSB, ADSB are displaced from SA or SB along [-1101] directions. $T$ and $O$ are the wide open interstitial positions along and parallel to the $c$-axis, while $\mathrm{HA}, \mathrm{HB}$ and $\mathrm{HAB}$ are "hexagonal" sites obtained by displacements from $\mathrm{O}$ sites parallel to the $c$-axis. The interstitial position of $\mathrm{Mg}$ identified in Ref. [60-Wahl 17] is indicated by the yellow circles. 
were used for the analysis, the derived ${ }^{27} \mathrm{Mg}$ displacement was slightly smaller, $+0.37-0.57 \AA$.

\subsection{Example 1: ${ }^{27} \mathrm{Mg}$ in $p$-GaN with the Timepix detector}

In this experiment $40 \mathrm{keV}{ }^{27} \mathrm{Mg}$ was implanted into a $\mathrm{GaN}$ single crystalline film at temperatures of $200^{\circ} \mathrm{C}, 600^{\circ} \mathrm{C}$ and $800^{\circ} \mathrm{C}$ while simultaneously measuring $\beta^{-}$emission channeling effects along the [-1101] direction. The experiment was performed for two different beam intensities, corresponding to implantation currents of $0.20 \mathrm{pA}$ and $1.4 \mathrm{pA}$ into a $1 \mathrm{~mm}$ diameter beam spot, acquiring about $10^{6}$ events per angular pattern (related implanted

${ }^{27} \mathrm{Mg}$ fluence $1.6 \times 10^{11} \mathrm{~cm}^{-2}$ ). The PSD used was a $3 \times 3 \mathrm{~cm}^{2}$ Timepix quad, which is an assembly of $2 \times 2$ Timepix chips, with a total of $512 \times 512$ pixels, mounted at a distance of 30 $\mathrm{cm}$ from the sample. The detector was operated with the pixelman software [62] in medipix mode with a frame rate of $0.2 \mathrm{~s}$. For the analysis by the maximum likelihood method the full pixel matrix of $512 \times 512$ pixels was used without summing over adjacent pixels. The $\sigma_{\text {conv }}$ value for the Gaussian smoothing was fixed at $0.05^{\circ}$ during the fit. Two sites were allowed in the fits: while the first site was kept fixed on the substitutional Ga position, the second, interstitial site was varied in small steps parallel to the $c$-axis. An example comparing measured and best fit patterns is shown in Fig. 5.

Fig. 6 shows for all implantation temperatures and currents the variation in absolute values of the cost function (negative log likelihood) when the position of the minority interstitial site is moved parallel to the $c$-axis, keeping the first site fixed at the substitutional Ga position. The best fits were achieved for interstitial positions close to the HA site, with the distances from O sites spreading from $+0.16 \AA$ to $+0.47 \AA$.

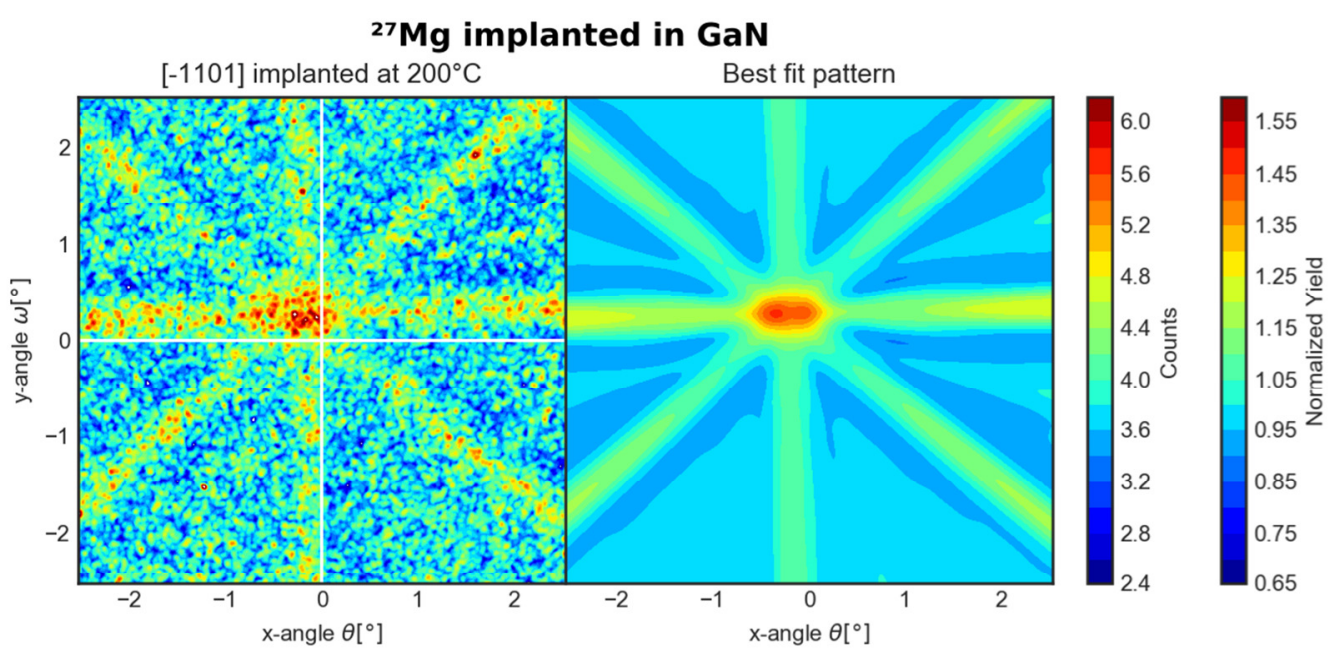

Fig. 5. Timepix [-1101] $\beta^{-}$emission pattern from ${ }^{27} \mathrm{Mg}$ in $p-\mathrm{GaN}$ and corresponding best fit. The measurement was performed for $0.20 \mathrm{pA}$ implantation at $200^{\circ} \mathrm{C}$ with the Timepix at 30 $\mathrm{cm}$ from the sample. The fitted pattern corresponds to $78 \%$ of ${ }^{27} \mathrm{Mg}$ on $\mathrm{S}_{\mathrm{Ga}}$ sites plus $22 \%$ on interstitial sites displaced by $+0.16 \AA$ from ideal O sites. Note that in the experimental pattern the white cross-shaped region represents the area along the edges of the four chips where no data are available. The colour bar titled "Counts" indicates the absolute number of counts per pixel, which was converted to the normalized yield according to eq. (12). Since the number of counts per pixel is very low (980000 events in the whole measurement, $\sim 3.7 /$ pixel), the original experimental data pattern has been slightly smoothed before displaying for better visualization as a pixel plot; the theoretical pattern is shown as a contour plot. 


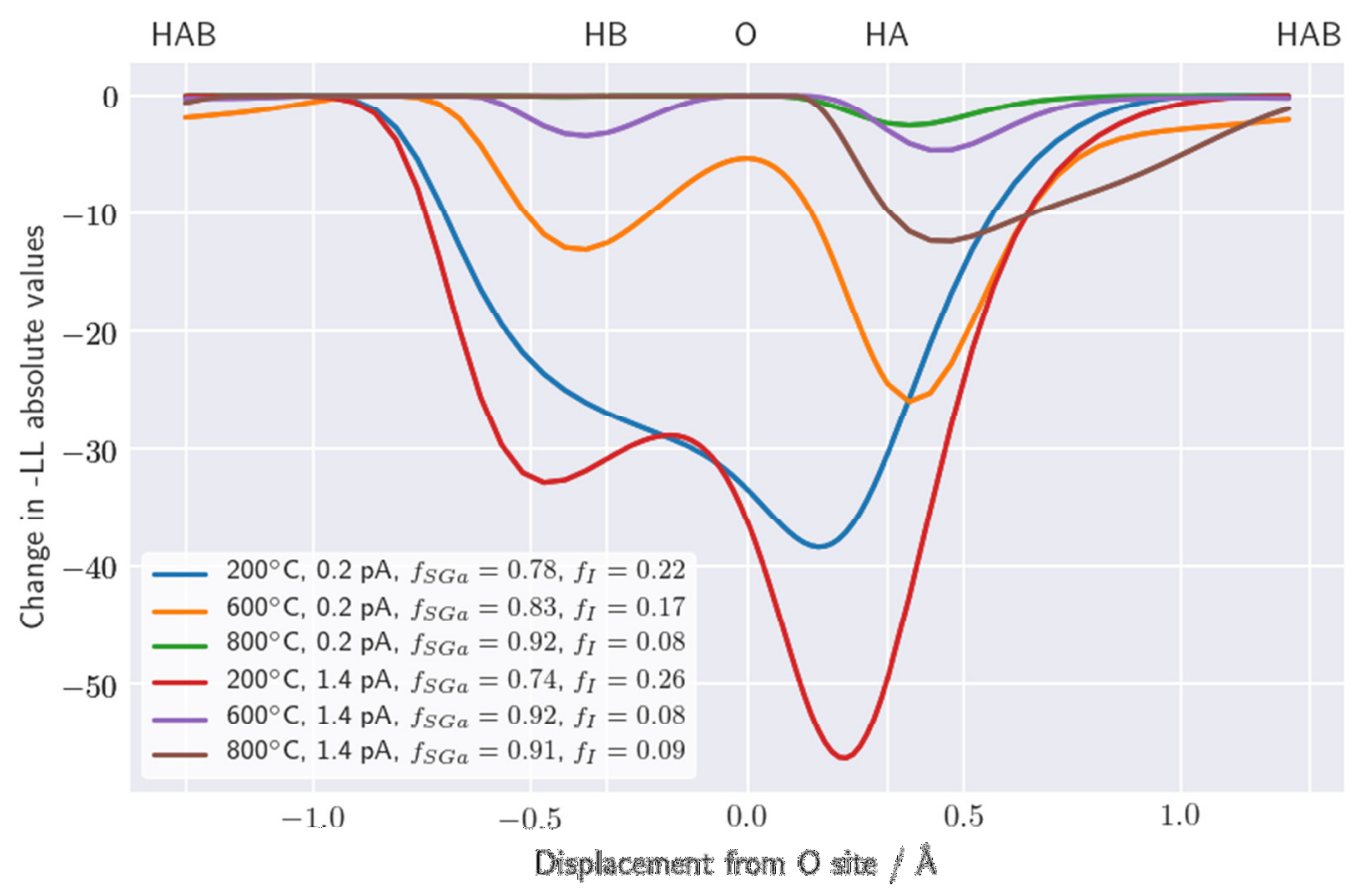

Fig. 6. Timepix fit results: change in the negative log likelihood (-LL) vs position of the second, interstitial ${ }^{27} \mathrm{Mg}$ site while keeping the first site fixed at the $\mathrm{S}_{\mathrm{Ga}}$ position. The cost function has two minima around the HA and HB site, with better results near HA. At higher implantation temperatures systematically less reductions in the $-\mathrm{LL}$ are observed due to the fact that the interstitial fraction of ${ }^{27} \mathrm{Mg}$ decreases (substitutional Ga $f_{\mathrm{SGa}}$ and interstitial $f_{\mathrm{I}}$ fractions at the LL minima are included in the legend).

\subsection{Example 2: ${ }^{27} \mathrm{Mg}$ in $p$-GaN with the pad detector}

In this case ${ }^{27} \mathrm{Mg}$ was implanted into $p$-GaN with $30 \mathrm{keV}$ at $27{ }^{\circ} \mathrm{C}, 300{ }^{\circ} \mathrm{C}$ and $450{ }^{\circ} \mathrm{C}$ with a beam current of $1.4 \mathrm{pA}$ into a $1 \mathrm{~mm}$ diameter spot. The measurements (Fig. 7) used a $3 \times 3$ $\mathrm{cm}^{2}$ pad PSD with $22 \times 22$ pixels, i.e. with the same surface area as the Timepix but mounted at twice the distance from the sample $(60 \mathrm{~cm})$. The fit procedure used was chi square minimization with $\sigma_{\text {conv }}$ for the Gaussian smoothing fixed at $0.05^{\circ}$ during the fit. Due to the large pixel size, the theoretically expected pixel yield was calculated as the average of an array of $6 \times 6$ simulated points inside the pixel, as explained in section 4.2.1. The pattern measured during implantation at $300{ }^{\circ} \mathrm{C}$ and the corresponding best fit can be seen in Fig. 7.

As in the first example, all patterns were fitted with the first site fixed at $S_{\mathrm{Ga}}$ while moving the second, interstitial site parallel to the $c$-axis. The reduction in chi-square as a function of the position of the interstitial site is shown in Fig. 8. Best fits were obtained for displacements from the $\mathrm{O}$ site of +0.37 to $+0.48 \AA$, i.e. also close to the HA site. Compared to the Timepix results, in these pad measurements a narrower spread of the positions of the interstitial site was obtained. However, this is probably not related to the different types of detectors and fitting procedures being used, but more likely due to the fact that, for these measurements, the pad detector was placed at twice the distance of the Timepix, thus providing a higher angular resolution that resolves more details around the axial effect. The chi-square obtained for the best fits (legend in Fig. 8) amounts to several times the number of degrees of freedom (number of pixels minus varied fit parameters). 


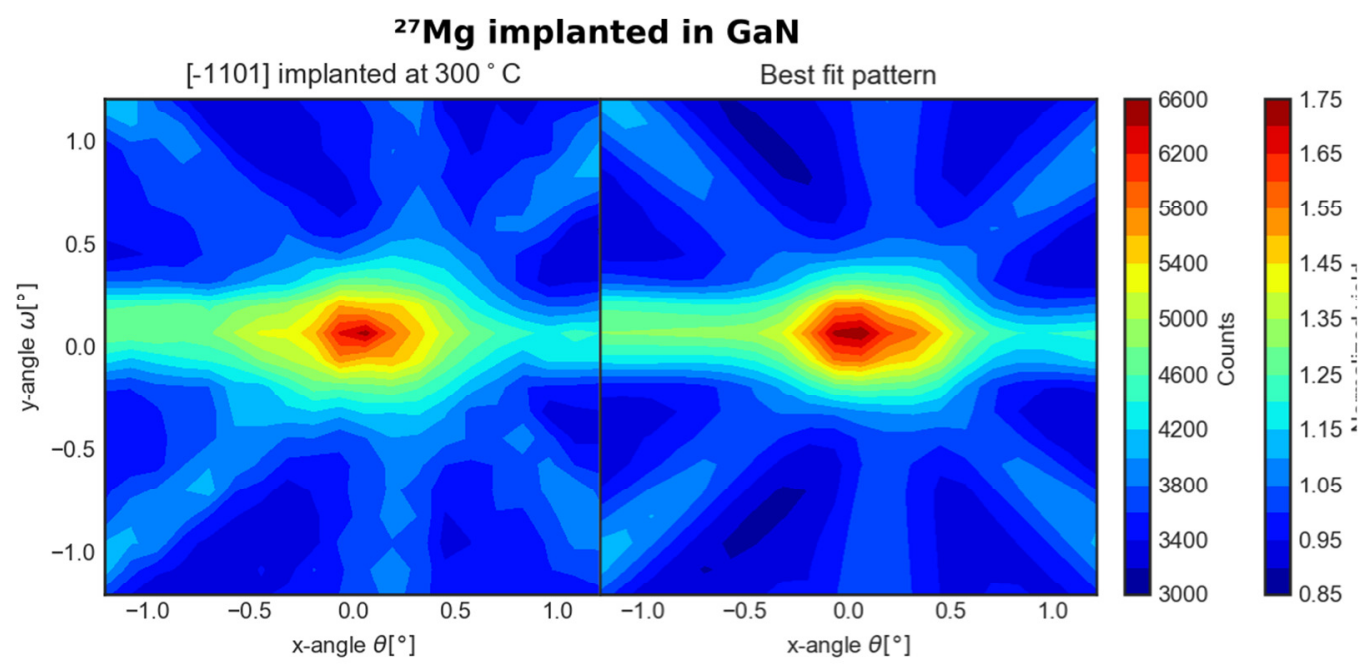

Fig. 7. Pad PSD [-1101] $\beta^{-}$emission pattern from ${ }^{27} \mathrm{Mg}$ in $p$-GaN and corresponding best fit as contour plots. The measurement was performed for $1.4 \mathrm{pA}$ implantation at $300^{\circ} \mathrm{C}$ with the PSD at $60 \mathrm{~cm}$ from the sample. The fitted pattern corresponds to $71 \%$ of ${ }^{27} \mathrm{Mg}$ on $\mathrm{S}_{\mathrm{Ga}}$ sites plus $29 \%$ on interstitial sites displaced by $+0.42 \AA$ from ideal O sites. The colour bar titled "Counts" represents the absolute number of counts per pixel (1400000 events in the whole pattern), which was converted to the normalized yield according to eq. (12). Note the smaller angular range of the pattern compared to the Timepix data in Fig. 5, due to twice the distance between detector and sample.

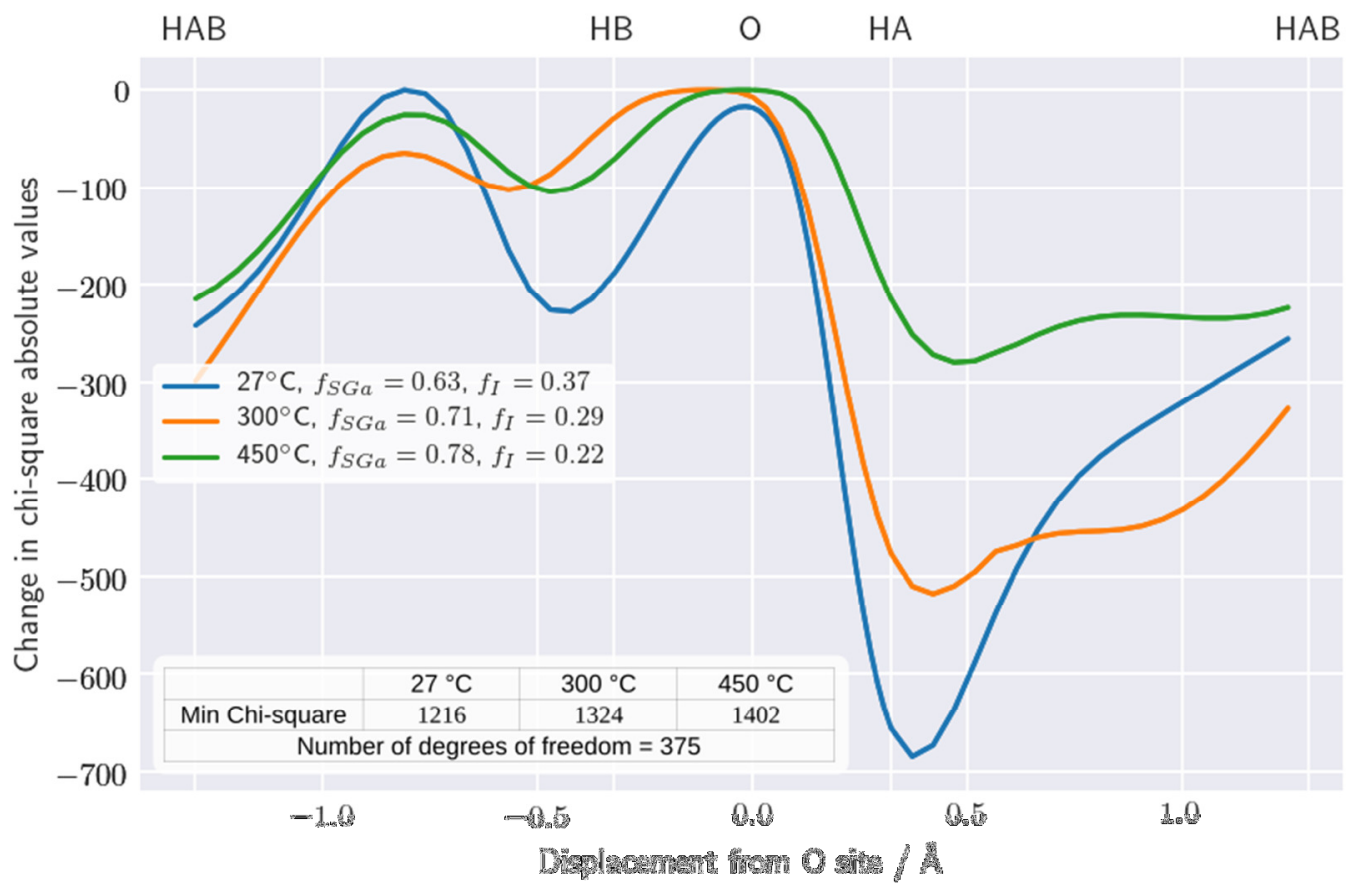

Fig. 8. Pad PSD fit results: absolute change in chi-square vs position of the second, interstitial ${ }^{27} \mathrm{Mg}$ site while keeping the first site fixed at the $\mathrm{S}_{\mathrm{Ga}}$ position. The greatest improvements in fit quality are obtained for interstitial sites near the HA position. Higher implantation temperatures result in smaller improvements of chi square since the interstitial fraction of ${ }^{27} \mathrm{Mg}$ decreases, as is mentioned in the legend. The absolute chisquare values corresponding to the three minima of the curves, as well as the number of degrees of freedom in the fits, are also shown. 


\subsection{Example 3: RBS/C from Ge}

The experimental data used in the third example consist of a ${ }^{4} \mathrm{He}$ RBS/C $2 \mathrm{D}$ image scan around the $<100>$ surface direction of a Ge single crystal. For that purpose the sample, mounted in a 3 -axis goniometer, was rotated in a matrix of $37 \times 37$ equidistant angular steps of $0.11^{\circ}$ by means of the M1 and M3 axes. At each angle the amount of $1.57 \mathrm{MeV}$ He particles backscattered under an angle of $167^{\circ}$ were recorded by an annular detector for a fixed number of channels that correspond to the depth range 0-1000 $\AA$ at random incidence and for a beam charge of $300 \mathrm{nC}(411 \mu \mathrm{C}$ in total). The He beam was collimated by two diaphragms of $3 \mathrm{~mm}$ and $1 \mathrm{~mm}$ spaced $260 \mathrm{~cm}$ apart, resulting in an angular beam spread of $\sim 0.09^{\circ}$. The experimental pattern is displayed in Fig. 9 on the left.

In order to produce theoretical patterns for the backscatter yield, FLUX simulations [49] were performed for an angular range of $\pm 3^{\circ}$ at equidistant steps of $0.025^{\circ}$ with 100000 projectiles per angle. A total of 11 simulations were run, each with a beam spread of $0^{\circ}$ but varying the rms displacement of the Ge atoms from $u_{1}(\mathrm{Ge})=0.059 \AA$ to $0.120 \AA$ in steps of $\sim 0.005 \AA$. The resulting patterns were then consecutively fitted to the experimental data by means of the PyFDD chi square approach, using in each case a fraction from the contribution of the perfect Ge crystal plus a fraction with a flat yield distribution (as from amorphous parts of the sample) and the angular beam spread $\sigma_{\text {conv }}$ as fit parameters. The simulated pattern which resulted in the best fit is displayed in Fig. 9 on the right and corresponds to a contribution of $88.8 \%$ from the Ge pattern with rms displacement of $u_{1}(\mathrm{Ge})=0.092 \AA$ measured with a beam spread of $\sigma_{\mathrm{conv}}=0.104^{\circ}$, plus an "amorphous" fraction of $11.2 \%$. While the angular spread corresponds quite well to what is expected from the collimation characteristics of the $\mathrm{He}$ beam, the best fit Ge rms displacement is somewhat higher than literature values [63] which scatter from $0.062 \AA$ to $0.087 \AA$. Together with the fact that the minimum yield around 0.166 was considerably higher than expected from a good single crystal (the simulations for $u_{1}(\mathrm{Ge})=0.092 \AA$ yielded 0.054$)$, this points towards the explanation that disorder was present

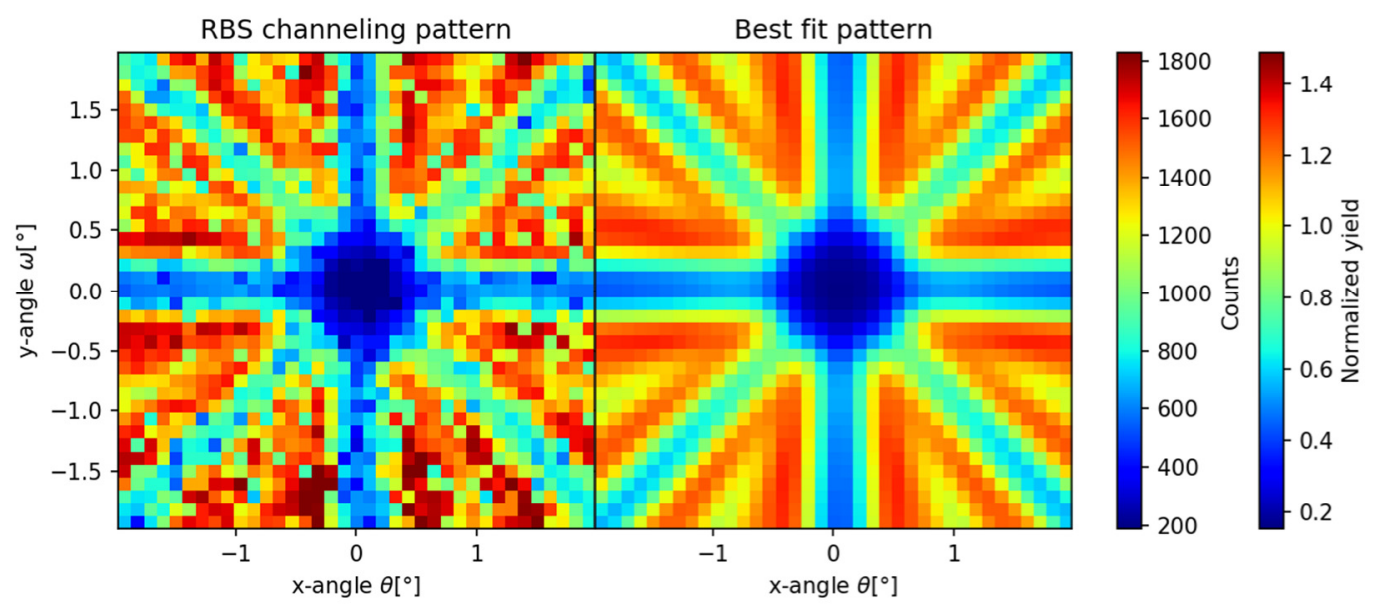

Fig. 9. RBS/C image scan of $1.57 \mathrm{MeV}{ }^{4} \mathrm{He}$ backscattering from $<100>\mathrm{Ge}$ (left) and corresponding best fit of FLUX simulations (right). The fitted pattern corresponds to $88.8 \%$ contribution from a perfect Ge crystal with $u_{1}(\mathrm{Ge})=0.092 \AA$ measured with a beam of angular spread $\sigma_{\text {conv }}=0.104^{\circ}$ and an amorphous fraction of $11.2 \%$, which were used as fit parameters. The colour bar titled "Counts" represents the absolute number of counts per angular position (1554135 events in the whole pattern), which was converted to the normalized yield according to eq. (12), thus resulting in a minimum yield of 0.166 for the fitted pattern. 
in the Ge crystal. This is also reflected by the amorphous fraction of $11.2 \%$ and is a consequence of the prolonged exposure to the analyzing beam during the acquisition of the image scan, which resulted in a cumulative fluence of $2.5 \times 10^{17} \mathrm{~cm}^{-2}$. It is known that a ${ }^{4} \mathrm{He}$ fluence of $3 \times 10^{17} \mathrm{~cm}^{-2}$ raises the minimum yield in Ge by a factor of 3-4 [64]. Thus, while the example shows how 2D channeling patterns can be used in order to characterize both the crystallographic rms displacements of host atoms and the angular resolution of an RBS/C setup, it also illustrates possible shortcomings of this approach due to effects from beam damage in radiation sensitive materials like Ge.

\section{Conclusions}

The use of two-dimensional channeling patterns is gaining more widespread use in many ion beam laboratories. However, except for emission channeling experiments, only in a small number of other cases such patterns have actually been used to extract quantitative information. We have described the theoretical framework for fitting 2D channeling patterns by the results of simulations, and its implementation in two software programs. The older, but fast FDD software is routinely used to analyze 2D patterns with high number of events per bin. For the newer software, called PyFDD, particular care was taken to make it better suitable for cases where the number of recorded events per bin is low, e.g. for measurements with high-resolution pixel detectors, or for RBS/C image scans for the location of small concentrations of foreign atoms. In order to fit such cases with the least possible bias, the chi square approach in PyFDD was improved, in particular by making the determination of the random level bias free. In addition, the possibility to perform maximum likelihood fits for extremely low numbers of events was implemented. As further development, PyFDD includes the option of performing an optimization of the Gaussian convolution of the theoretical patterns (which is related to the angular resolution) during the fit, which should be helpful in order to assess, e.g., changes in crystal quality in between various measurements (as a function of implantation fluence, annealing temperature etc).

The chi square and maximum likelihood approaches were compared for emission channeling experiments on the lattice location of ${ }^{27} \mathrm{Mg}$ in $p$-GaN, using measurements with a pad detector (several thousand counts per pad) and a Timepix high-resolution pixel detector (3-4 counts/pixel), leading to quite similar results. Finally, the usefulness of the 2D fit technique was also demonstrated by the analysis of an RBS/C image scan of a Ge crystal, demonstrating that by such measurements it is possible to extract at the same time information on the crystal quality, the rms displacement of hosts atoms, as well as the angular resolution of the setup.

\section{Acknowledgements}

We wish to acknowledge the support of the Portuguese Foundation for Science and Technology (FCT, projects CERN/FIS-PAR/0005/2017 and UID/Multi/04349/2019, PhD scholarship of E. David-Bosne SFRH/BD/95865/2013), of FWO Vlaanderen (PhD scholarships of T.A.L. Lima, grant numbers 29681 and 52152) and of KU Leuven (SF/18/008). The European Union's Horizon 2020 Framework research and innovation program supported ISOLDE beam times through grant agreement no. 654002 (ENSAR2) and use of KU Leuven facilities through grant agreement no. 824096 (RADIATE).

\section{References}

[1] W.K. Chu, J.W. Mayer, M.A. Nicolet, Backscattering spectrometry. Academic Press, New York, 1978.

[2] L.C. Feldman, J.W. Mayer, S.T. Picraux, Materials Analysis by Ion Channeling: Submicron Crystallography. Academic Press, New York, 1982. 
[3] A. Vantomme, 50 years of ion channeling in materials science, Nucl. Instr. Meth. Phys. Res. B 371 (2016) 12-26, http://dx.doi.org/10.1016/j.nimb.2015.11.035.

[4] M.D. Strathman, S. Baumann, Angle-resolved imaging of single-crystal materials with $\mathrm{MeV}$ helium ions, Nucl. Instr. Meth. Phys. Res. B 64 (1992) 840-845, http://dx.doi.org/10.1016/0168583X(92)95589-J.

[5] M.F. Wu, A. Vantomme, J. De Wachter, S. Degroote, H. Pattyn, G. Langouche, H. Bender, Comprehensive Rutherford backscattering and channeling study of ion-beam-synthesized $\mathrm{ErSi}_{1.7}$ layers, J. Appl. Phys. 79 (1996) 6920-6925, http://dx.doi.org/10.1063/1.361516.

[6] A. Mackova, V. Havranek, Ion beams provided by small accelerators for material synthesis and characterization, AIP Conf. Proc. 1852 (2017) 060003, http://dx.doi.org/10.1063/1.4984867.

[7] B. Brijs, W. De Coster, W. Vandervorst, Various advanced capabilities of the RBS setup at IMEC, Nucl. Instr. Meth. Phys. Res. B 64 (1992) 825-831, http://dx.doi.org/10.1016/0168-583X(92)95586G.

[8] M. Xu, Z. Atzmon, A. Schroer, B. Wilkens, J.W. Mayer, Visualization of ion channeling of (100) $\mathrm{Si}$ and $\mathrm{Si}_{1-x} \mathrm{Ge}_{x}$ epitaxial grown layers on silicon, Mater. Res. Soc. Symp. Proc. 316 (1994) 679, http://dx.doi.org/10.1557/PROC-316-679.

[9] L.T. Vinh, M. Eddrief, J.E. Mahan, A. Vantomme, J.H. Song, M.A. Nicolet, The van der Waals epitaxial growth of GaSe on Si(111), J. Appl. Phys. 81 (1997) 7289-7294, http://dx.doi.org/10.1063/1.365326.

[10] D.N. Jamieson, Structural and electrical characterisation of semiconductor materials using a nuclear microprobe, Nucl. Instr. Meth. Phys. Res. B 136-138 (1998) 1-13, http://dx.doi.org/10.1016/S0168-583X(97)00657-5.

[11] J. den Besten, D.N. Jamieson, C.G. Ryan, Lattice location of gold in natural pyrite crystals, Nucl. Instr. Meth. Phys. Res. B 152 (1999) 135-144, http://dx.doi.org/10.1016/S0168-583X(98)00965-3.

[12] D.J. Norris, M. Aindow, Measurement of epitaxial misorientations and related effects in thin films of $\mathrm{YBa}_{2} \mathrm{Cu}_{3} \mathrm{O}_{7-\delta}$ grown on nominally (001) $\mathrm{MgO}$ substrates by pulsed laser deposition, Thin Solid Films 423 (2003) 33-40, http://dx.doi.org/10.1016/S0040-6090(02)00999-9.

[13] A. Redondo-Cubero, K. Lorenz, N. Franco, S. Fernández-Garrido, R. Gago, P.J.M. Smulders , E Muñoz, E. Calleja, E. Alves, Influence of steering effects on strain detection in AlGaInN/GaN heterostructures by ion channeling, J. Phys. D: Appl. Phys. 42 (2009) 065420, http://dx.doi.org/10.1088/0022-3727/42/6/065420.

[14] X.M. Wang, K.B. Ma, D.N. Wijesundera, B. Liu, J.R. Liu, W.K. Chu, Azimuthal dependence and accurate determination of the half-angle in RBS channeling, Nucl. Instr. Meth. Phys. Res. B 267 (2009) 3466-3470, http://dx.doi.org/10.1016/j.nimb.2009.08.001.

[15] D. Wijesundera, K. Ma, X. Wang, B. Liu, J.R. Liu, W.K Chu, A geometric procedure for improved Rutherford backscattering channeling analysis of materials, Nucl. Instr. Meth. Phys. Res. B 267 (2009) 1948-1951, http://dx.doi.org/10.1016/j.nimb.2009.03.104.

[16] M. Madhuku, G. Husnain, I. Ahmad, H. Saleem, Irradiation-induced improvement in crystalline quality of epitaxially grown InGaN thin films: A preliminary study, Proceedings of SAIP2014, South African Institute of Physics, Lynnwood Ridge, 2014, p. 59-65.

[17] M. Usman, A. Hallén, A. Nazir, Ion implantation induced nitrogen defects in GaN, J. Phys. D: Appl. Phys. 48 (2015) 455107, http://dx.doi.org/10.1088/0022-3727/48/45/455107.

[18] E. Wendler, G. Becker, J. Rensberg, E. Schmidt, S. Wolf, W. Wesch, Direction-dependent RBS channelling studies in ion implanted $\mathrm{LiNbO}_{3}$, Nucl. Instr. Meth. Phys. Res. B 379 (2016) 195-199, http://dx.doi.org/10.1016/j.nimb.2016.03.007. 
[19] K. Jin, H. Bei, Y. Zhang, Ion irradiation induced defect evolution in Ni and Ni-based FCC equiatomic binary alloys, J. Nucl. Mater. 471 (2016) 193-199, http://dx.doi.org/10.1016/j.jnucmat.2015.09.009.

[20] M. Erich, M. Kokkoris, S. Fazinic, S. Petrovic, EBS/C proton spectra from a virgin diamond crystal, Nucl. Instr. Meth. Phys. Res. B 381 (2016) 96-102, http://dx.doi.org/10.1016/j.nimb.2016.05.030.

[21] M. Wang, A. Debernardi, Y. Berencén, R. Heller, C. Xu, Y. Yuan, Y. Xie, R. Böttger, L. Rebohle, W. Skorupa, M. Helm, S. Prucnal, S. Zhou, Breaking the doping limit in silicon by deep $\begin{array}{llllll}\text { impurities, } & \text { Phys. } & \text { Rev. } & \text { Appl. } & 11 & \text { (2019) }\end{array}$ http://dx.doi.org/10.1103/PhysRevApplied.11.054039.

[22] J. De Wachter, P. Hendrickx, H. Pattyn, G. Langouche, B. Brijs, W. Vandervorst, Channeling detection using position sensitive detectors, Nucl. Instr. Meth. Phys. Res. B 79 (1993) 829-831, http://dx.doi.org/10.1016/0168-583X(93)95479-O.

[23] H. Ellmer, R. Aichinger, E. Winkler, D. Semrad, V. Mergel, O. Jagutzki, Crystal adjustment by means of blocking pattern, Rev. Sci. Instr. 71 (2000) 2693-2697, http://dx.doi.org/10.1063/1.1150676.

[24] P.A. Miranda, U. Wahl, N. Catarino, M. Ribeiro da Silva, E. Alves, Performance of resistivecharge position sensitive detectors for RBS/Channeling applications, Nucl. Instr. Meth. Phys. Res. A 760 (2014) 98-106, http://dx.doi.org/10.1016/j.nima.2014.05.062.

[25] A. Redondo-Cubero, E. David-Bosne, U. Wahl, P. Miranda, M.R. da Silva, J.G. Correia, K. Lorenz, Strain detection in crystalline heterostructures using bidimensional blocking patterns of channeled particles, J. Phys. D: Applied Physics 51 (2018) 115304, http://dx.doi.org/10.1088/13616463/aaad8b.

[26] S. Shimoda, T. Kobayashi, Development of three-dimensional medium-energy ion scattering spectroscopy, Nucl. Instr. Meth. Phys. Res. B 219-220 (2004) 573-577, http://dx.doi.org/10.1016/j.nimb.2006.04.066.

[27] S. Shimoda, T. Kobayashi, Structure of an Er silicide film on a Si(111) substrate using threedimensional medium-energy ion scattering, J. Appl. Phys. 96 (2004) 3550-3552, http://dx.doi.org/10.1063/1.1778820.

[28] T. Kobayashi, Development of three-dimensional medium-energy ion scattering using a large solid angle detector, Nucl. Instr. Meth. Phys. Res. B 249 (2006) 266-269, http://dx.doi.org/10.1016/j.nimb.2004.01.122.

[29] M.K. Linnarsson, A. Hallén, J. Åström, D. Primetzhofer, S. Legendre, G. Possnert, New beam line for time-of-flight medium energy ion scattering with large area position sensitive detector, Rev. Sci. Instr. 83 (2012) 095107, http://dx.doi.org/10.1063/1.4750195.

[30] H. Trombini, P.L. Grande, A. Hentz, M. Vos, A. Winkelmann, A comparison of the analysis of non-centrosymmetric materials based on ion and electron beams, Nucl. Instr. Meth. Phys. Res. B 431 (2018) 31-37, http://dx.doi.org/10.1016/j.nimb.2018.06.026.

[31] W. Assmann, H. Huber, C. Steinhausen, M. Dobler, H. Glückler, A. Weidinger, Elastic recoil detection analysis with heavy ions, Nucl. Instr. Meth. Phys. Res. B 89 (1994) 131-139, http://dx.doi.org/10.1016/0168-583X(94)95159-4.

[32] H. Nolte, W. Assmann, H. Huber, S. A. Karamian, H. D. Mieskes, Blocking- and channelingERDA with heavy ions, Nucl. Instr. Meth. Phys. Res. B 136-138 (1998) 587-593, http://dx.doi.org/10.1016/S0168-583X(97)00824-0.

[33] H. Hofsäss, G. Lindner: Emission channeling and blocking, Physics Reports 201, (1991) 121183, http://dx.doi.org/10.1016/0370-1573(91)90121-2. 
[34] H. Hofsäss, S. Winter, S. G. Jahn, U. Wahl, E. Recknagel, Emission channeling studies in semiconductors, Nucl. Instr. Meth. Phys. Res. 63 (1992) 83-90, http://dx.doi.org/10.1016/0168$\underline{583 X(92) 95174-\mathrm{P}}$.

[35] U. Wahl, ISOLDE collaboration, Emission channeling studies of Li in semiconductors, Physics Reports 280 (1997) 145, http://dx.doi.org/10.1016/S0370-1573(96)00021-X.

[36] U. Wahl, Advances in electron emission channeling measurements in semiconductors" Hyperfine Interactions (2000) 129 349-370, http://dx.doi.org/10.1023/A:1012697429920.

[37] U. Wahl, J.G. Correia, A. Costa, E. David-Bosne, L.M.C. Pereira, L.M. Amorim, V. Augustyns, K. Temst, A. Vantomme, M.R. da Silva, D.J. Silva, J.P. Araújo, P. Miranda, K. Bharuth-Ram, Emission Channeling with Short-Lived Isotopes (EC-SLI) at CERN's ISOLDE Facility, in Exotic Nuclei, pp. 563-573, WORLD SCIENTIFIC, Sept. 2014, http://dx.doi.org/10.1142/9789814632041_0061.

[38] U. Wahl, S.G. Jahn, M. Restle, C. Ronning, H. Quintel, K. Bharuth-Ram, ISOLDE collaboration, Alpha-emission channeling investigations of the lattice location of $\mathrm{Li}$ in $\mathrm{Ge}$, Nucl. Instr. Meth. Phys. Res. B 118 (1996) 76-81, http://dx.doi.org/10.1016/0168-583X(95)01196-X.

[39] U. Wahl, J.G. Correia, S. Cardoso, J.G. Marques, A. Vantomme, G. Langouche, Electron emission channeling with position-sensitive detectors, Nucl. Instr. Meth. Phys. Res. B 524 (2004) 245-256, http://dx.doi.org/10.1016/S0168-583X(97)00768-4.

[40] U. Wahl, J.G. Correia, A. Czermak, S.G. Jahn, P. Jalocha, J.G. Marques, A. Rudge, F. Schopper, J.C. Soares, A. Vantomme, P. Weilhammer, Position-sensitive Si pad detectors for electron emission channeling experiments, Nucl. Instr. Meth. Phys. Res. A 524 (2004) 245-256, http://dx.doi.org/10.1016/j.nima.2003.12.044.

[41] A.C. Marques, U. Wahl, J.G. Correia, M.R. Silva, A. Rudge, P. Weilhammer, J.C. Soares, Noise and trigger efficiency characterization of cooled silicon pad detectors, 524 A 572 (2007) 1056-1064, http://dx.doi.org/10.1016/j.nima.2006.12.028.

[42] X. Llopart, R. Ballabriga, M. Campbell, L. Tlustos, W. Wong, Timepix, a 65k programmable pixel readout chip for arrival time, energy and/or photon counting measurements, Nucl. Instr. Meth. Phys. Res. A 581 (2007) 485-494, http://dx.doi.org/10.1016/j.nima.2007.08.079.

[43] M. Campbell, 10 years of the Medipix2 Collaboration, Nucl. Instr. Meth. Phys. Res. A 633 (2011) S1-S10, http://dx.doi.org/10.1016/j.nima.2010.06.106.

[44] E. David-Bosne, PyFDD version 0.7.0, Zenodo, Github-, (2019) http://dx.doi.org/10.5281/zenodo.3247499.

[45] R. Catherall, W. Andreazza, M. Breitenfeldt, A. Dorsival, G.J. Focker, T.P. Gharsa, T.J. Giles, J.L. Grenard, F. Locci, P. Martins, S. Marzari, J. Schipper, A. Shornikov, T. Stora, The ISOLDE facility, J. Phys. G: Nucl. Particle Phys. 44 (2017) 094002, http://dx.doi.org/10.1088/13616471/aa7eba.

[46] K. Johnston, J. Schell, J.G. Correia, M. Deicher, H.P. Gunnlaugsson, A.S. Fenta, E. DavidBosne, A.R.G. Costa, D.C. Lupascu, The solid state physics programme at ISOLDE: recent developments and perspectives, J. Phys. G: Nucl. Particle Phys. 44 (2017) 104001, http://dx.doi.org/10.1088/1361-6471/aa81ac.

[47] M.R. Silva, U. Wahl, J. G. Correia, L.M. Amorim, L.M.C. Pereira, A versatile apparatus for online emission channeling experiments, Rev. Sci. Instr. 84 (2013) 073506, http://dx.doi.org/10.1063/1.4813266.

[48] B. De Vries, U. Wahl, S. Ruffenach, O. Briot, A. Vantomme, Influence of crystal mosaicity on axial channeling effects and lattice site determination of impurities, Appl. Phys. Lett. 103 (2013) 172108, http://dx.doi.org/10.1063/1.4826705.

[49] P. Smulders, D. Boerma, Computer simulation of channeling in single crystals, Nucl. Instr. Meth. Phys. Res. B 29 (1987) 471-489, http://dx.doi.org/10.1016/0168-583X(87)90058-9. 
[50] J. Lindhard, Influence of crystal lattice on motion of energetic charged particles, Mat. Fys. Medd. Dan. Vid. Selsk. 34 (1965), 1-64.

[51] J.F. Ziegler, M.D. Ziegler, J.P. Biersack, SRIM - The stopping and range of ions in matter (2010), Nucl. Instr. Meth. Phys. Res. B 268 (2010) 1818-1823, http://dx.doi.org/10.1016/j.nimb.2010.02.091.

[52] G. Cowan, Statistical Data Analysis, Clarendon Press, Oxford, New York, 1998.

[53] B. De Vries, Lattice site location of impurities in group III nitrides using emission channeling, $\mathrm{PhD}$ thesis, KU Leuven, 2006, http://cds.cern.ch/record/1640588.

[54] L.M. Pinto de Almeida Amorim, Lattice site location of electrical dopant impurities in group-III nitrides, PhD thesis, KU Leuven, 2016, http://cds.cern.ch/record/2240696.

[55] W.H. Press, B.P. Flannery, S.A. Teukolsky, W.T. Vetterling, Numerical Recipes, Cambridge University Press, Cambridge, 1986.

[56] S. Baker, R. D. Cousins, Clarification of the use of chi-square and likelihood functions in fits to histograms, Nucl. Instr. Meth. Phys. Res. 221 (1984) 437-442, http://dx.doi.org/10.1016/01675087(84)90016-4.

[57] B. Roe, Chi-square fitting when overall normalization is a fit parameter, arXiv:1506.09077 (2015), http://arxiv.org/abs/1506.09077.

[58] E. Jones, E. Oliphant, P. Peterson, et al, SciPy: Open Source Scientific Tools for Python, 2001, http://www.scipy.org [Online; accessed 2019-08-09].

[59] S. Agostinelli, J. Allison, K. Amako et al, Geant4 - a simulation toolkit, Nucl. Instr. Meth. Phys. Res. B 506 (2003) 250-303, http://dx.doi.org/10.1016/S0168-9002(03)01368-8.

[60] S. Brandt, Data Analysis: Statistical and Computational Methods for Scientists and Engineers, Springer, Berlin, 2014.

[61] U. Wahl, L. Amorim, V. Augustyns, A. Costa, E. David-Bosne, T. Lima, G. Lippertz, J. Correia, M. da Silva, M. Kappers, K. Temst, A. Vantomme, L. Pereira, Lattice Location of Mg in GaN: a fresh look at doping limitations, Phys. Rev. Lett. 118 (2017) 095501, http://dx.doi.org/10.1103/PhysRevLett.118.095501.

[62] D. Turecek, T. Holy, J. Jakubek, S. Pospisil, Z. Vykydal, Pixelman: a multi-platform data acquisition and processing software package for Medipix2, Timepix and Medipix3 detectors, J. Instrumentation 6 (2011) C01046, http://dx.doi.org/10.1088/1748-0221/6/01/C01046.

[63] Landolt-Börnstein - Group III Condensed Matter (Numerical Data and Functional Relationships in Science and Technology), Group IV Elements, IV-IV and III-V Compounds, Part a - Lattice Properties, vol 41A1a, eds. O. Madelung, U. Rössler, and M. Schulz, Springer, Berlin, Heidelberg, 2001, http://dx.doi.org/10.1007/b60136.

[64] D. Benzeggouta, I. Vickridge (eds.), Handbook on best practice for minimising beam induced damage during IBA, arXiv:1303.3171 (2013), https://arxiv.org/abs/1303.3171v1. 